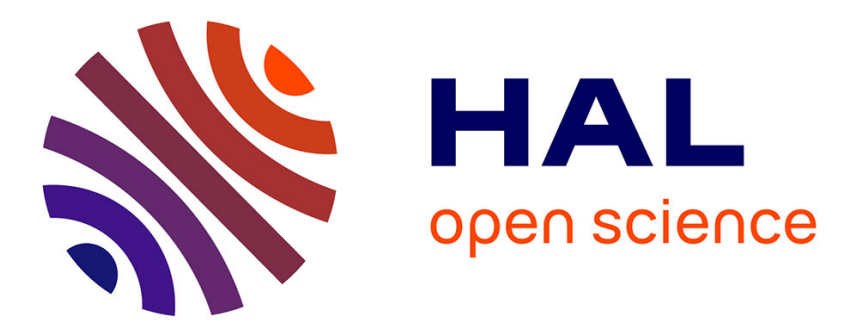

\title{
Trajectoires perçues. Une approche quantitative des perceptions de bien-être
}

Eva Lelièvre, Nicolas Robette

\section{To cite this version:}

Eva Lelièvre, Nicolas Robette. Trajectoires perçues. Une approche quantitative des perceptions de bien-être. Bulletin de Méthodologie Sociologique / Bulletin of Sociological Methodology, 2015, 125, pp.25-45. 10.1177/0759106314543754 . halshs-01143596

\section{HAL Id: halshs-01143596 https://shs.hal.science/halshs-01143596}

Submitted on 18 Apr 2015

HAL is a multi-disciplinary open access archive for the deposit and dissemination of scientific research documents, whether they are published or not. The documents may come from teaching and research institutions in France or abroad, or from public or private research centers.
L'archive ouverte pluridisciplinaire HAL, est destinée au dépôt et à la diffusion de documents scientifiques de niveau recherche, publiés ou non, émanant des établissements d'enseignement et de recherche français ou étrangers, des laboratoires publics ou privés. 


\title{
Trajectoires perçues \\ Une approche quantitative des perceptions de bien-être
}

\author{
par \\ Éva Lelièvre ${ }^{1}$ \\ (INED) \\ Nicolas Robette \\ (Printemps-CNRS-UVSQ, Ined)
}

\begin{abstract}
In the Biographies and entourage (2001) survey, the life-event histories of 2,830 respondents were collected. In addition to their family, residential and occupational histories, each respondent was asked to identify and explain the successive significant periods, turning points and general tone of their life course and what they viewed as landmark events. We therefore deal with a well-being trajectory made of the successive perceived tones of each period. We undertake a descriptive analysis of these trajectories and turning points: the division of life into periods reveals patterns in terms of the age distribution of turning points and of their coincidence with factual events. From a methodological point of view, taking such an approach which combines facts and perceptions contributes to the questioning of the boundaries between qualitative and quantitative approaches.
\end{abstract}

\section{Résumé}

Dans l'enquête par questionnaire Biographies et entourage (Ined, 2001), la reconstitution par les 2830 enquêtés des événements factuels composant leurs trajectoires familiale, résidentielle et professionnelle a été enrichie d'une synthèse plus personnelle de la biographie. Chacun s'est ainsi vu proposer de découper sa vie, en identifiant et en décrivant brièvement les périodes signifiantes, les tournants biographiques et les événements considérés comme marquants. Les enquêtés recomposent alors un enchaînement de périodes aux tonalités variées : une trajectoire de vie perçue/subjective. L'analyse de ces trajectoires révèle des régularités en termes de répartition des tournants biographiques et coïncidence entre événements factuels et perceptions. Ainsi l'analyse conjointe des dimensions perçues et factuelles redessine les frontières entre démarches qualitative et quantitative.

\section{Keywords}

Perception, well-being, life course, turning point

\section{Mots clefs}

Perception, bien-être, trajectoire, biographie, tournant biographique

Les collectes biographiques qualitatives comme quantitatives sollicitent la reconstitution d'une «histoire» qui est relatée de façon narrative (Lelièvre et Vivier, 2001-2009). L'élaboration des outils de collecte et leur adaptation aux différents contextes et aux

\footnotetext{
${ }^{1}$ Corresponding Author :

Eva Lelièvre, INED, 133 boulevard Davout, 75020, Paris, France

Email : eva@ined.fr
} 
questionnements spécifiques se sont de fait déroulées dans un dialogue pluridisciplinaire (GRAB, 2010) qui a favorisé un mode de questionnement alternatif au jeu des questionsréponses, suscitant un discours proche de celui qui est produit dans le processus de remémoration. Ce mode de collecte, plus adapté au processus de narration spontanée, associe et organise les événements biographiques les uns par rapport aux autres («j'ai changé de travail un an avant mon arrivée à Paris $»)$ et permet de prendre en compte allers retours et ajustements au fil de l'entretien (« en fait, mon mariage, c'est au moment du décès de mon père, ensuite ma mère nous a rejoint »). Plus qu'avec d'autres sources quantitatives, les échanges pluridisciplinaires et les travaux sur l'approche biographique ont largement discuté de l'illusion objectiviste $\mathrm{du}$ recueil quantitatif factuel (GRAB, 2006). Les travaux méthodologiques menés autour des collectes biographiques rétrospectives mais aussi prospectives depuis les années 1980 (Courgeau, 1985; Poulain et al., 1991; GRAB, 1999: 5973) ont montré la fiabilité des données recueillies avec des outils adaptés du point de vue de la cohérence chronologique des parcours (Courgeau et Lelièvre, 1989: 19-21) et de leur complétude (Mazuy et Lelièvre, 2005). Si ces travaux confirment la présence d'une dimension subjective à cette reconstruction, ils soulignent également le fait qu'il faut en tirer parti. Ainsi, prenant acte de ce « flou », l'enquête Biographie et entourage a introduit de façon systématique le recueil des perceptions que les répondants ont de leur trajectoire, des événements qui l'ont marquée et des différentes périodes qui la composent (Lelièvre et al., 2006; Bonvalet et Lelièvre, 2012). On a ici affaire à une approche qui intègre, au sein d'un même questionnement et avec un même outil de collecte, des démarches quantitative et qualitative qui reconstruisent conjointement des faits et des perceptions. C'est à partir de ce matériau que nous proposons ici une analyse de la perception des trajectoires biographiques.

\section{Perceptions de bien-être, du qualitatif au quantitatif}

Dans ce dossier consacré à l'articulation du quantitatif et du qualitatif au sein des trajectoires, nos travaux portent plus précisément sur la prise en compte d'éléments de perception qui ne sont pas issus d'une collecte qualitative. En effet, les expériences de mesure du bien-être subjectif (Ferriss, 2001; Haller et Müller, 2008) à partir d'enquêtes auprès des ménages ont montré que l'approche était pertinente tant en économie - où l'attribution en 2002 du prix Nobel d'économie à Daniel Kahneman pour ses travaux dans ce domaine montre que ce champ de recherche est à la fois considéré comme une sous-discipline légitime et qu'elle n'est somme toute pas si récente - qu'en santé publique, où le bien-être est plutôt exploré dans sa forme restreinte à la santé subjective. Dans ces spécialités, les indicateurs de bien-être ont fait preuve de leur robustesse et sont couramment utilisés (Amiel et al., 2013; Jylhä, 2009). En démographie, la collecte des motivations et l'analyse de la causalité des processus traduisent une préoccupation de compréhension des comportements analysés ${ }^{2}$.

Dans le cas des collectes biographiques rétrospectives, la combinaison des points forts des deux approches : la reconstitution factuelle des trajectoires individuelles et collectives, accompagnée d'une mesure de bien-être facile à collecter, est prometteuse, comme l'ont montré les premiers travaux exploitant ce type de matériaux (Lelièvre et al., 2006; Laborde et al., 2007; Golaz et Lelièvre, 2012).

\section{Collecter des trajectoires perçues}

\footnotetext{
2 Ainsi l'exploration du désir d'enfant constitue un des champs de l'étude de la fécondité (Régnier-Loilier, 2007).
} 
Nous utilisons les données de l'enquête Biographies et entourage (2001), une collecte rétrospective conduite auprès de 2830 Franciliens nés de 1930 à 1950. Le questionnaire, rempli lors d'un entretien en face à face, aborde successivement les trajectoires familiale et professionnelle des membres de l'entourage de l'enquêté, puis les jalons résidentiels, professionnels et familiaux de sa propre trajectoire, année après année, portés sur une grille biographique (Figure 1). Enfin, une synthèse libre est faite par l'enquêté à la fin de l'entretien au vu des éléments factuels de la grille. Le répondant est ainsi amené à découper sa vie en différentes périodes dont il place les frontières temporelles sur la même grille. Ces périodes sont caractérisées les unes par rapport aux autres. Un premier découpage est fait du point de vue de son bien-être, un autre de celui de l'aisance financière des ménages auquel il/elle appartient tout au long de sa vie.

Les deux premières parties s'appuient donc sur un questionnement essentiellement fermé et factuel, tandis que les synthèses, qui terminent l'entretien, fournissent une interprétation du déroulement, des temps forts et de la tonalité générale de la biographie selon le propre regard de l'enquêté (Lelièvre et Vivier, 2001; Laborde et al., 2007). Un élément important de ces appréciations est également le fait, que pour l'ensemble des enquêtés âgés de 50 à 70 ans, ils établissent ce découpage avec un recul comparable vis-à-vis de la constitution de leur famille et du déroulement de leur vie active. Ce regard porté sur l'ensemble de la trajectoire (plus de 50 ans) permet aux enquêtés d'en identifier les inflexions et de qualifier les séquences qui la composent, en modulant leurs appréciations pour donner une cohérence d'ensemble qui tienne compte des dimensions familiale, professionnelle et historique. Cette mise en perspective du parcours factuel et de son appréciation correspond bien à la démarche préconisée par T. Hareven et K. Masaoka (1988) pour la recherche des tournants biographiques. Elle est systématisée auprès d'enquêtés interviewés tard dans leur parcours. En effet, y compris pour un même individu, l'interprétation proposée est susceptible d'évoluer au fil du temps : des événements marquants perdent de leur importance après quelques années alors que d'autres plus anodins peuvent, avec le recul, apparaître comme de véritables tournants. Il est donc assez primordial pour leur comparabilité de traiter ces appréciations produites par des enquêtés ayant un recul suffisant et comparable.

Ces données ont fait l'objet d'un premier article (Laborde et al., 2007) centré sur les événements marquants et une description du découpage des trajectoires. Dans le présent travail, nous proposons tout d'abord d'analyser plus avant les tournants biographiques ${ }^{3}$ qui séparent les périodes perçues, leur distribution par âge et leur association avec des jalons factuels, puis de rendre compte de l'enchaînement des périodes et des trajectoires de bien-être qu'elles dessinent en construisant une typologie des trajectoires perçues ${ }^{4}$.

\footnotetext{
${ }^{3}$ Nous n'interrogeons pas ici l'imprévisibilité et l'irréversibilité de ces moments des biographies, contrairement à l'étude des «bifurcations » (voir par exemple Grossetti, 2006). Nous les envisageons simplement comme inflexions, i.e. connexions de trajectoires stables à l'intérieur des parcours de vie, dans la continuité de travaux sur les «turning points » (Hareven et Masaoka, 1988 ; Abbott, 1997), à partir du témoignage des enquêtés euxmêmes et non de l'interprétation a posteriori du chercheur.

${ }^{4}$ Seules les questions Sy1 et Sy2 seront analysées. Les événements marquants (Sy3) font l'objet d'un autre article (Laborde et al., 2007).
} 


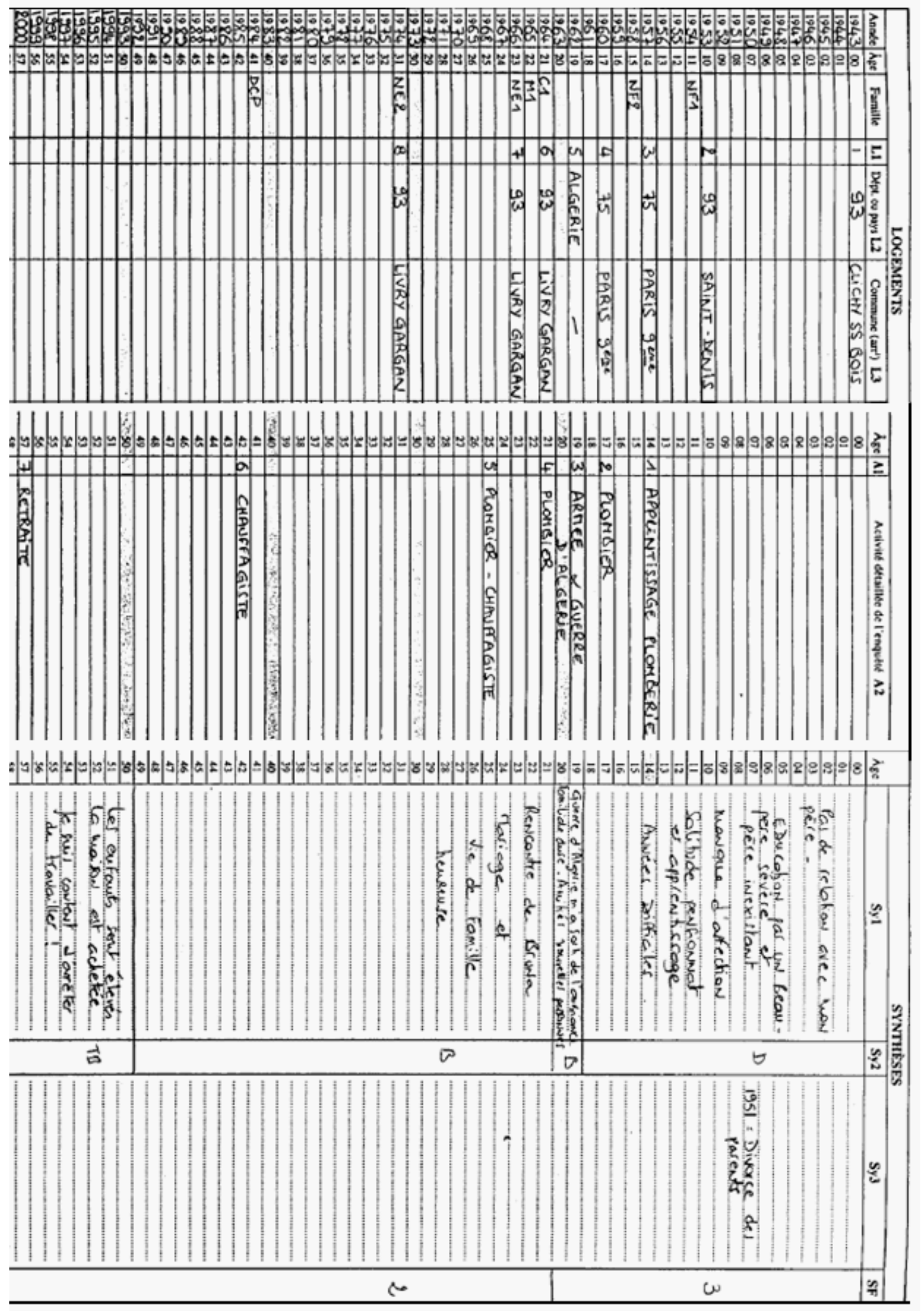

Lecture: Pour ce chauffagiste qui vient de prendre sa retraite, on a reproduit ici les jalons de sa trajectoire familiale en première colonne (naissance de ses deux demi-frères, mise en couple mariage, naissance de ses deux enfants et décès de son père), de sa trajectoire résidentielle et professionnelle qui sont mis en regard de son découpage suscité par les questions suivantes :

Sy1 - Pouvez-vous découper votre vie en époques différentes ? Caractérisez ces périodes, en particulier, en identifiant ce qui les différencie les unes des autres et ce qu'elles représentent dans votre vie.

Sy2 - Pour chacune de ces époques, était-ce : 


\begin{tabular}{|c|} 
TB de très bonnes années \\
B de bonnes années \\
SP des années sans problème \\
D des années difficiles \\
TD des années très difficiles \\
Sy3 - Y a-t-il des événements personnels ou historiques qui ont marqué votre vie? \\
SF - Echelle d'appréciation des revenus du ménage
\end{tabular}

Figure 1. Exemple abrégé de recueil factuel et perçu de l'enquête Biographies et entourage (Ined, 2001)

\section{Les changements de période déclarés à l'épreuve des jalons factuels}

\section{« Découper » son parcours de vie}

L'interprétation par les enquêtés de leur propre trajectoire fait émerger des représentations variées, notamment en terme de nombre de périodes citées. Cependant, très peu d'individus ne souhaitent pas du tout découper leur trajectoire, considérant que leur vie « est un long fleuve tranquille ». A l'opposé, moins de 1 pourcent distinguent dix périodes ou plus. Le nombre médian de périodes est de quatre et deux tiers des enquêtés mentionnent de trois à cinq périodes (Laborde et al., 2007).

Une régression logistique modélisant la probabilité de citer plus de périodes que la moyenne (Laborde et al., 2007) fait apparaître le poids du capital scolaire dans cet exercice d'interprétation : les personnes plus diplômées déclarent plus de périodes. Les trajectoires résidentielles et professionnelles semblent très liées à la perception de la trajectoire biographique, puisque plus les étapes qui les scandent sont nombreuses, plus le nombre de périodes perçues déclarées croît. Les résultats sont plus nuancés en ce qui concerne l'histoire familiale : le nombre d'enfants ou le décès de proches n'ont pas d'effet significatif, au contraire des divorces, séparations ou veuvages. Mais l'absence d'effet dans le modèle ne signifie pas nécessairement que les événements concernés ne marquent pas souvent un changement de période. En effet, il se peut par exemple que la naissance d'un premier enfant soit perçue comme un changement de période chez une très large part des enquêtés, que ceux-ci déclarent beaucoup de périodes ou non.

Plus de 8000 changements de période désignés par les 2830 enquêtés se répartissent tout au long de leur parcours (Figure 2). Loin d'être uniforme, cette répartition présente un profil caractéristique : les tournants sont très peu nombreux dans les premières années de la vie, puis leur nombre croît fortement jusqu'à atteindre un pic vers l'âge de vingt ans. Leur fréquence diminue ensuite jusqu'aux environs de trente ans, et reste relativement constante jusqu'à cinquante ans. 


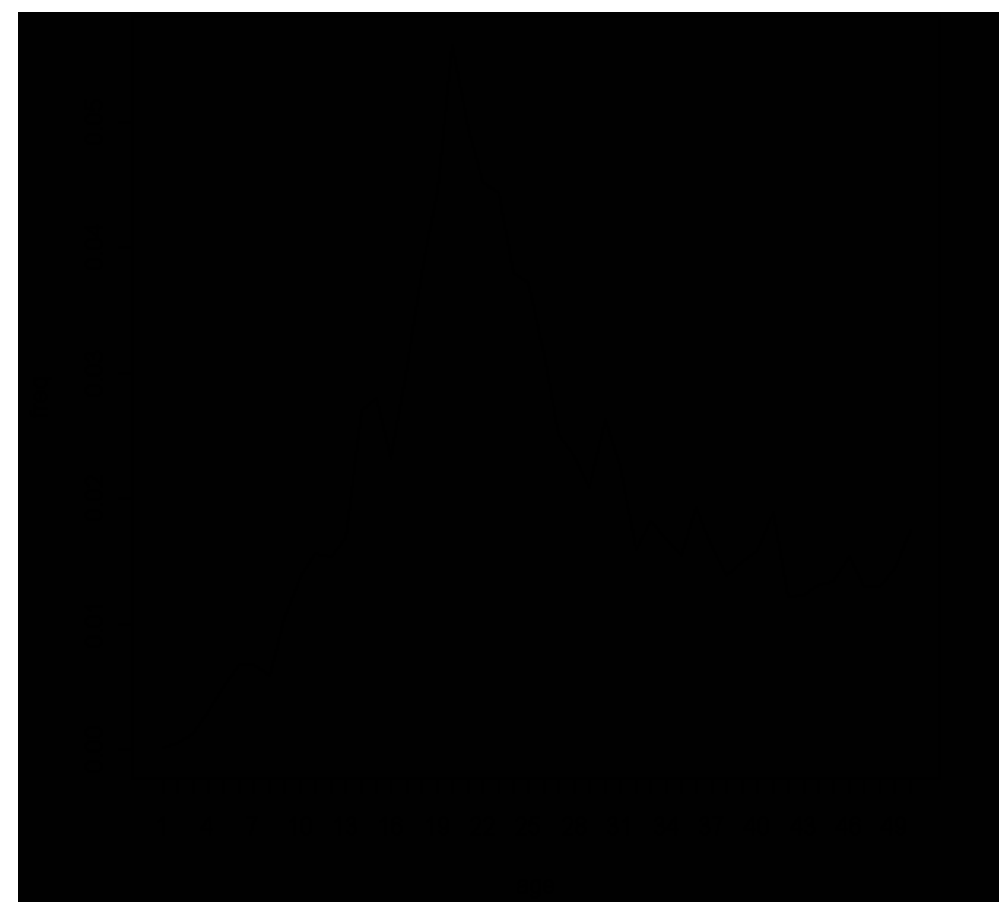

Figure 2. Distribution par âge des changements de période perçus

Par ailleurs, lorsque l'on examine cette distribution selon le sexe, la génération, le diplôme, l'origine sociale ou géographique, le profil de la répartition par âge des tournants désignés reste remarquablement stable et proche de celle que l'on vient de décrire : sa forme générale et le pic vers vingt ans semblent être des caractéristiques qui traversent l'ensemble des groupes sociaux.

\section{Jalons factuels et tournants perçus}

Pour aller plus loin dans l'exploration de la relation entre jalons factuels et tournants dans la trajectoire perçue, nous allons donc maintenant les confronter systématiquement, en calculant la part des jalons factuels d'un type donné (par exemple, les changements de logement) qui coïncident (à un an près) avec un tournant dans la trajectoire perçue (Tableau 1).

Les événements familiaux jouent ici un rôle prépondérant. Ainsi, 44 pourcent des mariages et 43 pourcent des mises en couple sont associés à un tournant. Le rang de l'événement est déterminant : les proportions sont de 47 pourcent pour les premiers mariages comme pour les premières mises en couple, contre respectivement 21 pourcent et 29 pourcent pour les unions de rang supérieur. Puis viennent le décès d'un conjoint (34 pourcent coïncident avec un changement de période), les séparations ou divorces ( 25 pourcent) et les décès d'un enfant ( 24 pourcent). Les naissances d'un enfant marquent moins souvent un tournant (17 pourcent des premières naissances, 6 pourcent des suivantes), de même que les événements affectant les parents des enquêtés.

Tableau 1. Coïncidence entre jalons factuels et tournants biographiques

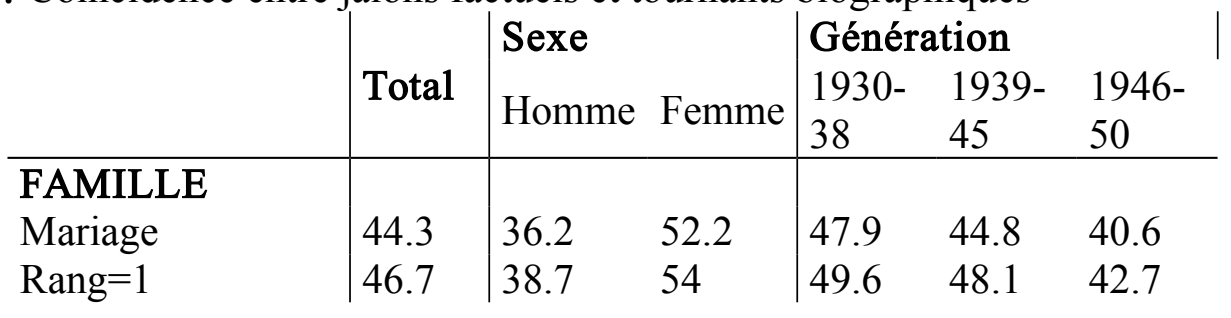




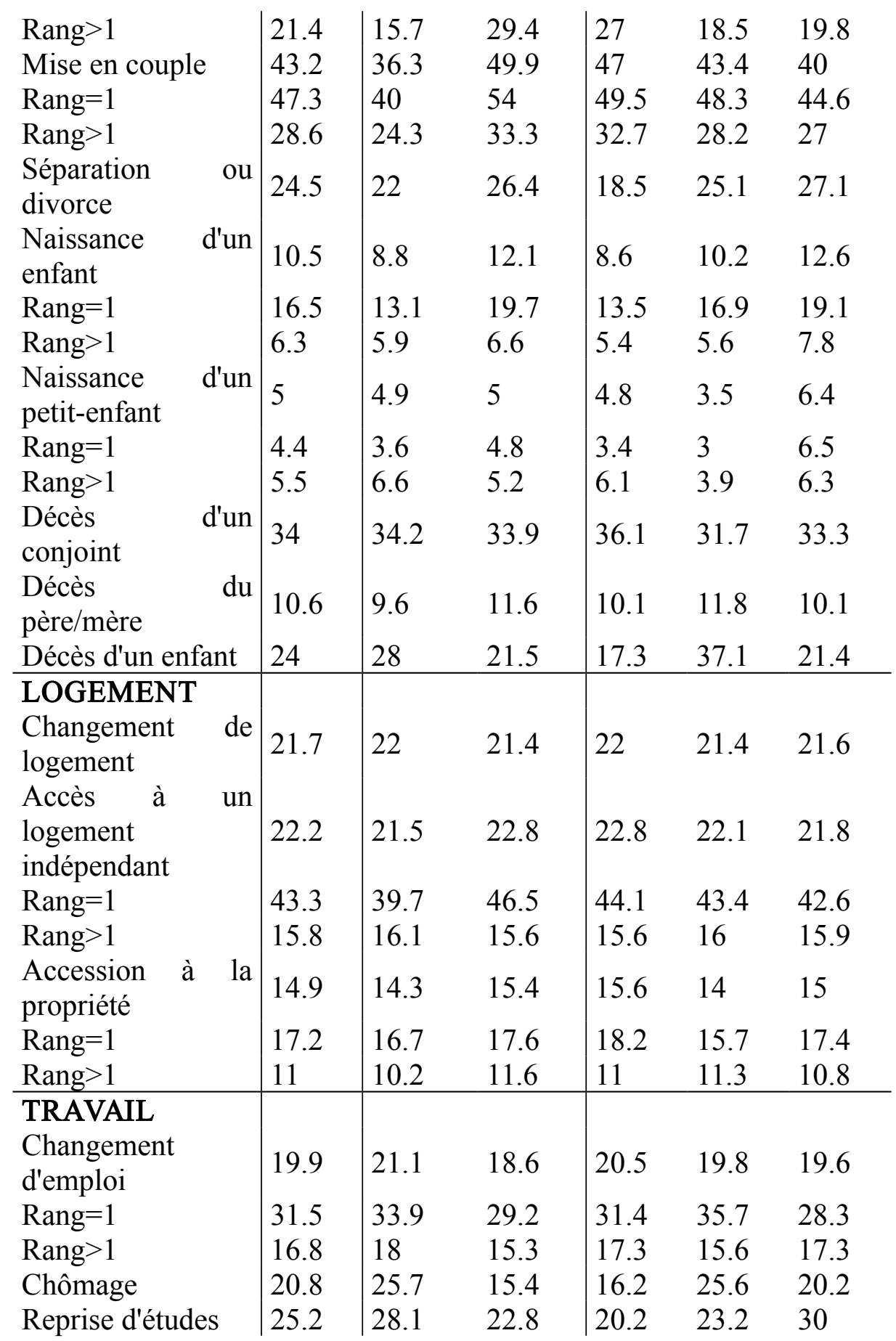

Source : INED, Enquête « Biographies et entourage », 2001.

Champ : Ensemble des enquêtés ayant répondu à la question $(\mathrm{N}=2799$ femmes et hommes Franciliens âgés de 50 à 70 ans).

Lecture : 44,3 pourcent des mariages coïncident (à un an près) avec un tournant biographique.

S'agissant des événements de la trajectoire professionnelle, 20 pourcent des changements d'emploi sont concomitants d'un tournant biographique (32 pourcent lorsqu'il s'agit de l'accès à un premier emploi), 21 pourcent des entrées au chômage et 25 pourcent des reprises d'études, un événement rare qui marque souvent une véritable réorientation professionnelle. Dans la sphère résidentielle, 22 pourcent des changements de logement coïncident avec un tournant, mais 43 pourcent des accès à un premier logement indépendant et seulement 15 pourcent des accessions à la propriété (17 pourcent des primo-accessions). Au final, on remarque notamment l'importance de quelques seuils classiques du passage à l'âge adulte, en 
particulier la première mise en couple, le premier logement indépendant et le premier emploi, ce qui n'est sans doute pas sans lien avec le pic observé vers vingt ans dans la distribution par âge des tournants biographiques. Dans des travaux précédents (XX pas de référence Tichit et Lelièvre, 2006), nous avons discuté du repérage du "passage à l'âge adulte» dans les parcours et montré qu'il consiste en un moment d'accumulation événementielle de "premières fois" (précédé et suivi de périodes de stabilité), auquel le plus souvent est également associé le découpage de la biographie que l'enquêté a proposé en synthèse, signalant un tournant biographique investi de sens par l'enquêté, qui conjugue ainsi informations factuelles et subjectives.

Sans surprise, les résultats diffèrent sensiblement pour les hommes et les femmes, autour d'une opposition famille / travail. Les événements familiaux sont plus souvent associés à des tournants par les femmes (54 pourcent vs 39 pourcent pour les premières mises en couple, 20 pourcent vs 13 pourcent pour les premières naissances, 31 pourcent vs 21 pourcent des séparations, par exemple). A l'inverse, les événements professionnels coïncident plus souvent avec un tournant chez les hommes (34 pourcent vs 29 pourcent des premiers emplois, 26 pourcent vs 15 pourcent des épisodes de chômage, 28 pourcent vs 23 pourcent des reprises d'études). De façon intéressante, l'accès à un premier logement indépendant semble plus marquant pour les femmes que pour les hommes (47 pourcent vs 40 pourcent).

On observe par ailleurs en reflet, l'évolution des structures et valeurs familiales au fil des cohortes : l'importance croissante (bien que relativement limitée) accordée aux enfants, puisque la part des premières naissances associées à un tournant passe de 13 pourcent à 19 pourcent entre les générations 1930-1938 et 1946-1950, et aux séparations et divorces (de 18 pourcent à 27 pourcent), alors que dans le même temps les mises en couple passent de 47 pourcent à 40 pourcent au fur et à mesure que la cohabitation prémaritale rend le début des unions moins aisément identifiable.

L'opposition entre sphère familiale et professionnelle structure également les différences entre milieux sociaux (voir annexe 1). En effet, les personnes qui appartiennent au groupe professionnel des cadres et professions intellectuelles supérieures au moment de l'enquête sont celles qui associent le moins souvent des événements familiaux à des tournants biographiques - qu'il s'agisse des mises en couples, des séparations ou de la naissance du premier enfant - de même que l'accès à un premier logement indépendant. A l'inverse, le premier emploi coïncide plus souvent pour elles avec un tournant. On observe, à grands traits, un profil opposé à l'autre pôle de l'espace social, même si ce constat doit être nuancé, principalement $\mathrm{du}$ fait de la forte spécialisation sexuée des catégories d'employés et d'ouvriers.

\section{Le déroulement des trajectoires}

\section{La tonalité des trajectoires}

Au-delà du découpage dont nous venons d'apprécier les facteurs structurants, s'ouvre l'autre pan de l'analyse : celui de la tonalité des différentes périodes identifiées par les enquêtés, et de l'enchaînement de ces périodes. En effet, chacune d'entre elles est qualifiée selon la directive suivante : Pour chacune de ces époques, était-ce? De très bonnes années TB, de bonnes années $\mathrm{B}$, des années sans problème $\mathrm{SP}$, des années difficiles $\mathrm{D}$, des années très difficiles TD. La description initiale des qualificatifs attribués aux périodes qui composent les trajectoires de la naissance jusqu'à 50 à 70 ans (selon l'âge de l'enquêté au moment de la collecte) montre que les périodes d'appréciation positive (bonnes et très bonnes années) sont majoritaires (57 pourcent), qu'elles concernent 37 années soit les deux tiers de la durée 
moyenne des trajectoires (58 années) et qu'elles se répartissent sans polarisation sur une période en particulier ${ }^{5}$ (Laborde et al., 2007). Globalement, pour les individus enquêtés, 20 pourcent des périodes déclarées ont été qualifiées d'années difficiles (respectivement 8 pourcent très difficiles) et elles représentent au total 8,7 années (respectivement 3 années). Les périodes plus neutres (années sans problème) constituent 15 pourcent de l'ensemble pour une durée de 9 années et demie.

Les enquêtés avaient en outre la possibilité de décrire plus précisément les périodes (Figure 1, colonne Sy1). Cette caractérisation des périodes permet de mieux comprendre ce que recouvrent les appréciations synthétiques (Difficile, Sans Problème, etc. en colonne Sy2). Pour traiter les commentaires que font les enquêtés sur les périodes, nous avons procédé à une analyse textuelle. On réalise initialement une lemmatisation ${ }^{6}$ de ces données (regroupement des mots de la même famille, verbes et leur formes conjuguées, etc.), puis une analyse lexicométrique $\mathrm{du}$ corpus: on repère simplement les mots les plus significativement surreprésentés pour un type d'appréciation synthétique donné (D/TD ; SP ; B/TB) et un âge donné (selon que les périodes se déroulent au moins en partie avant 20 ans; entre 20 et 30 ans; après $30 \mathrm{ans}^{7}$ ). Le tableau 2 détaille par époques les mots qui caractérisent les qualificatifs respectifs ${ }^{8}$.

Tableau 2. Les mots les plus représentatifs des périodes, selon leur tonalité et l'âge où elles se déroulent

\begin{tabular}{|c|c|c|c|c|}
\hline & \multicolumn{3}{|l|}{ Appréciation de la période } \\
\hline & & TD-D & SP & B-TB \\
\hline \multirow[t]{2}{*}{ Âge } & $\begin{array}{l}\text { Avant } \\
20 \\
\text { ans }\end{array}$ & $\begin{array}{l}\text { - Guerre, conflit, exode } \\
\text { - Père, mère, parents } \\
\text { - Misère, pauvreté, } \\
\text { manque, manger, faim, } \\
\text { nourriture, froid, } \\
\text { souffrance, peur } \\
\text { - Pension, sévérité }\end{array}$ & $\begin{array}{l}\text { - Enfance, souvenir, } \\
\text { normal, adolescence, } \\
\text { école, parents }\end{array}$ & $\begin{array}{l}\text { - Protection, cocon, } \\
\text { chaleureux, entourage, } \\
\text { gâté-e, parents, grands- } \\
\text { parents } \\
\text { - Insouciance, s'amuser, } \\
\text { heureux } \\
\text { - Études, école } \\
\text { - Campagne }\end{array}$ \\
\hline & $\begin{array}{l}\text { Entre } \\
20 \text { et } \\
30 \\
\text { ans }\end{array}$ & $\begin{array}{l}\text { - Algérie, service militaire } \\
\text { - Mariage } \\
\text { - Logement } \\
\text { - Alcoolisme }\end{array}$ & $\begin{array}{l}\text { - Mariage, couple } \\
\text { - Service militaire }\end{array}$ & $\begin{array}{l}\text { - Mariage, couple, } \\
\text { rencontre, famille, enfant, } \\
\text { naissance } \\
\text { - Célibat, indépendance, } \\
\text { voyage } \\
\text { - Profession, travail, } \\
\text { carrière, réussite } \\
\text { - Construire, responsabilité }\end{array}$ \\
\hline
\end{tabular}

${ }^{5}$ On n'observe pas, par exemple, d'enfance systématiquement idyllique.

${ }^{6}$ La lemmatisation a été réalisée au moyen des logiciels Alceste et Spad-T.

${ }^{7}$ Ce découpage correspond à grands traits à la succession enfance / passage à l'âge adulte / vie adulte pour ces générations.

${ }^{8}$ Les commentaires qui suivent constituent une première exploration des données textuelles, qui sera prolongée dans des travaux ultérieurs. 


\begin{tabular}{|c|c|c|c|}
\hline $\begin{array}{l}\text { Aprè } \\
\mathrm{s} \quad 30 \\
\text { ans }\end{array}$ & $\begin{array}{l}\text { - Santé, malade, handicap, } \\
\text { accident, moral, } \\
\text { dépression } \\
\text { - Chômage, licenciement } \\
\text { - Divorce, séparation, } \\
\text { solitude, décès, mort }\end{array}$ & $\begin{array}{l}\text { - Retraite } \\
\text { - Routine, calme, aller } \\
\text { mieux }\end{array}$ & $\begin{array}{l}\text { - Sérénité, stabilité, calme, } \\
\text { équilibre, épanouissement, } \\
\text { satisfaction, profiter } \\
\text { - Activité, voyage, } \\
\text { bricolage, bénévolat, } \\
\text { association, organiser, } \\
\text { consacrer } \\
\text { - Nouveau, reprendre, } \\
\text { retrouver, mieux } \\
\text { - Petits-enfants } \\
\text { - Profession }\end{array}$ \\
\hline
\end{tabular}

Source : INED, Enquête « Biographies et entourage », 2001.

Champ : Ensemble des enquêtés ayant répondu à la question $(\mathrm{N}=2799$ femmes et hommes Franciliens âgés de 50 à 70 ans).

Lecture : «guerre », «conflit» ou « exode» figurent parmi les mots les plus sur-représentés dans la caractérisation des périodes difficiles ou très difficiles se déroulant (au moins en partie) avant 20 ans.

Du fait des générations enquêtées, la guerre est très présente dans les périodes difficiles situées avant 20 ans. Les conditions de vie sont aussi au cœur des récits, où la misère et la pauvreté s'associent au manque de nourriture et au froid. Dans certains cas, un placement en pension et/ou une éducation sévère constituent aussi des souvenirs pénibles qui colorent une période particulière de la trajectoire biographique. Une précédente analyse des moments de séparation de la famille dans l'enfance et l'adolescence avait d'ailleurs montré une grande concordance avec la perception exprimée de périodes difficiles voire très difficiles (Golaz et Lelièvre, 2012). A l'inverse, les périodes de l'enfance que les enquêtés qualifient de bonnes (B-TB) sont associées à la présence d'un entourage (parents et grands-parents, notamment) protecteur et chaleureux, les études y sont vécues positivement et la tonalité générale est celle de l'insouciance et du bonheur.

Chez les jeunes adultes, les caractérisations des périodes difficiles font souvent référence à la sphère domestique ("mariage », "logement»), sans que l'on puisse aller beaucoup plus avant dans l'analyse, et - pour les hommes - à l'armée (" service militaire », "Algérie »). L'alcoolisme est aussi relativement fréquemment mentionné. C'est la construction de la famille d'élection qui domine les périodes positives : la rencontre d'un partenaire, la mise en couple et/ou le mariage et la naissance des enfants. Mais le célibat est aussi parfois valorisé dans sa dimension d'autonomie. La sphère professionnelle compte également parmi les éléments perçus comme positifs, e.g. la réussite de la carrière.

Après 30 ans, les périodes difficiles se caractérisent souvent par des problèmes de santé, physique ou mentale, et par la solitude, que celle-ci soit le fait de séparations ou du décès de membres de l'entourage. La sphère professionnelle est aussi citée, avec les licenciements et le chômage. Les périodes positives, elles, sont caractérisées principalement par la sérénité ressentie par les enquêtés (" calme », "stabilité », " équilibre ») et les plaisirs qu'elle procure (« épanouissement», « satisfaction»). Cet épanouissement se traduit par des activités (« voyage », « bricolage», « bénévolat») nouvelles ou retrouvées (« reprendre », « retrouver ») et par la présence des petits-enfants.

Les périodes considérées comme "sans problème » donnent lieu à des descriptions généralement plus courtes et peu détaillées.

De cette description globale de la caractérisation des périodes ressort le fait que la tonalité « de bonnes années » domine en durée dans les trajectoires. De plus, si l'on procède à une analyse textuelle de la description des périodes faite par les enquêtés, un vocabulaire

\footnotetext{
${ }^{9}$ On est ici de nouveau en présence d'un effet générationnel.
} 
spécifique apparaît selon la tonalité et l'époque de la vie auquel les répondants se réfèrent. Pour poursuivre l'analyse, il convient à présent d'examiner l'enchainement des périodes au sein des trajectoires individuelles des déclarations de bien-être.

\section{Les trajectoires individuelles de bien-être perçu}

Une première façon de décrire les trajectoires de bien-être dans leur ensemble est d'envisager la succession des qualificatifs donnés aux périodes. Les trajectoires qui alternent au moins deux tonalités et décrivent "des hauts et des bas » constituent la grande majorité (78 pourcent), qui se déclinent en un très grand nombre de combinaisons possibles. Du côté de la stabilité, 18 pourcent des parcours sont qualifiés de Bien et/ou Très Bien uniquement; à l'inverse, seulement 1 pourcent des trajectoires sont intégralement négatives (Difficiles ou Très Difficiles). Pour parvenir à cette description rapide, il a été nécessaire de procéder à une simplification, en regroupant les périodes décrites comme de bonnes et très bonnes années d'un part, et des années difficiles et très difficiles d'autre part, ce qui revient à " gommer » l'intensité des évaluations pour ne retenir que les tonalités positive, négative ou neutre. En dépit de cette réduction drastique de l'information, le constat de la diversité s'impose, comme le montre le tableau 3. En effet, si l'on retient les trajectoires perçues correspondant à au moins 2,5 pourcent des individus, on ne décrit que 40 pourcent de l'ensemble des trajectoires. La recherche de régularité dans ces parcours, mêmes résumés, s'avère donc difficile et nécessite d'avoir recours à des techniques plus élaborées.

Tableau 3. Une description simplifiée des trajectoires les plus fréquemment citées (regroupant au moins 2,5 pourcent des parcours)

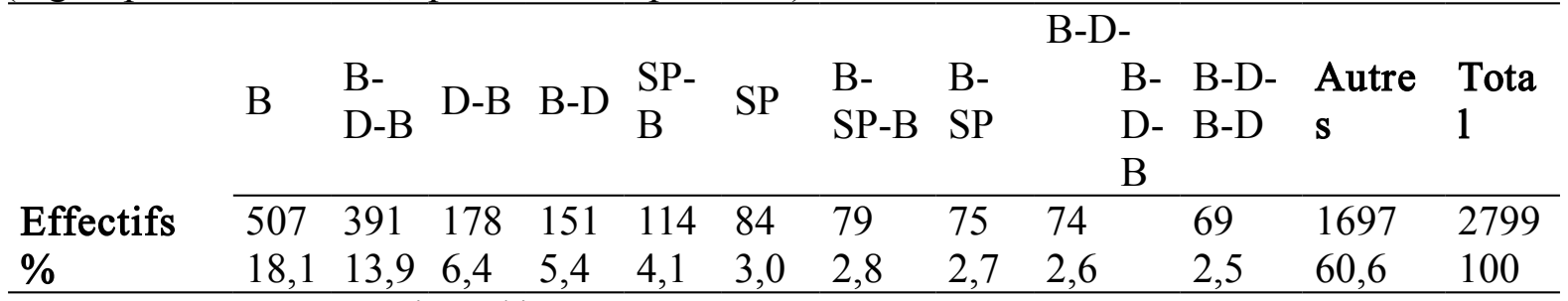

Source : INED, Enquête « Biographies et entourage », 2001.

Champ : Ensemble des enquêtés ayant répondu à la question $(\mathrm{N}=2799$ femmes et hommes Franciliens âgés de 50 à 70 ans).

Lecture : 13,9 pourcent des Franciliens âgés de 50 à 70 ans ont eu une trajectoire de vie BDB. Bien et Très Bien sont regroupés en « $\mathrm{B}$ », Difficile et Très Difficile en « $\mathrm{D} »$.

On souhaite en effet prendre en compte la succession, la durée et la tonalité des périodes qui s'enchaînent et qui, d'un individu à l'autre, sont d'un nombre variable. Pour décrire synthétiquement les trajectoires entières dans leur complexité et sans éliminer une partie du corpus, nous utilisons donc les méthodes d'analyse de séquences, qui permettent de repérer les régularités de l'évolution des perceptions au long des parcours de vie en identifiant un nombre limité de trajectoires-type. La technique retenue est l'Optimal Matching (ou appariement optimal, voir annexe 2). Cette méthode présente l'avantage de prendre en compte conjointement les différentes dimensions temporelles des séquences, c'est-à-dire le moment auquel interviennent les transitions, la durée et l'ordre des étapes (Robette, 2011). Les trajectoires perçues sont donc codées sous forme de séquences avec un élément par année, chaque élément pouvant prendre la valeur TD, D, SP, B ou TB. Pour l'ensemble des trajectoires collectées, on ne considère que les 50 premières années afin de traiter des séquences de même longueur. 


\section{De douze à cinq types de trajectoires de bien-être perçu}

Lorsque l'on soumet la matrice de distances entre les 2799 trajectoires perçues, issue de l'algorithme d'Optimal Matching, à une classification ascendante hiérarchique (avec le critère d'agrégation de Ward), le choix de deux partitions en un nombre élevé puis réduit de classes 12 et 5 classes, qui « expliquent » respectivement 63 pourcent et 47 pourcent de l'information - permet de discuter finement des régularités observées. La classification ascendante hiérarchique aboutit en effet à un arbre de classification, appelé dendrogramme, dont chaque niveau correspond à une partition de l'ensemble des individus (Figure 2). Le choix du nombre de classes n'obéit pas à un critère statistique ${ }^{10}$ mais plutôt à la volonté d'obtenir des classes pertinentes d'un point de vue théorique et homogènes, tout en résumant suffisamment la diversité de l'échantillon des trajectoires pour en faciliter la description.

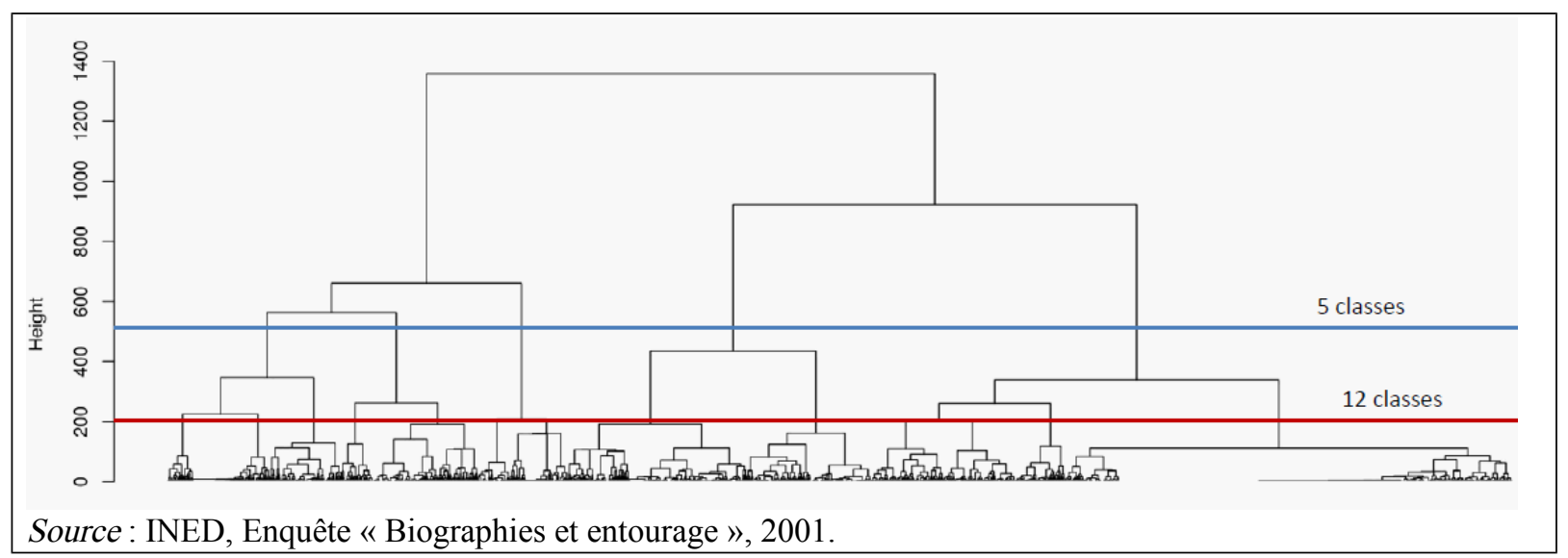

Figure 1. Dendrogramme de la classification des trajectoires de bien-être

Le premier découpage en 12 classes, dont nous ne commenterons que la répartition, nous permet d'examiner les principales régularités, qui correspondent souvent à des trajectoires stables, mais aussi des parcours plus rares mais ayant des profils intéressants, avec des changements de tonalité. Le tableau 4 présente les classes de la typologie selon l'enchainement temporel des tonalités, ainsi que leur représentation graphique (voir explication à propos de la figure 3). Sans surprise, la tonalité « Bonnes années » domine, ainsi que nous l'avions identifié précédemment, mais des nuances apparaissent qui distinguent des trajectoires où la vie adulte présente des difficultés passagères (classe 2) ou plus durables mais d'intensité variable (classes 4 et 5), ou encore tardivement dans la quarantaine (classe 3).

Les profils sans problèmes et qui s'améliorent rassemblent 15 pourcent des trajectoires (classes 6 et 7), puis viennent les parcours à l'enfance difficile mais qui s'améliorent (classe 8 $=13$ pourcent) et les vies plus globalement jugée pénibles (classe 9).

Tableau 4. Partition en 12 classes de trajectoires de bien-être à partir d'une analyse de séquences

\begin{tabular}{llll}
\hline numéro & parangon & $N$ & $\%$ \\
\hline 1 & B & 932 & $33,3 \%$
\end{tabular}

\footnotetext{
${ }^{10}$ Même si les sauts d'inertie observés sur le dendrogramme permettent de guider le choix.
} 
Dominante

B $\quad 300 \quad 10,7 \%$

étape D

3

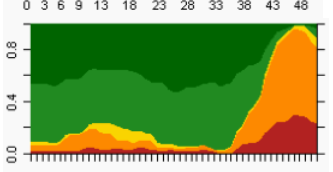

$B$ à D

tardivement

104

$3,7 \%$

4

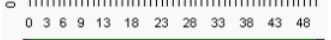

$\mathrm{B}$ à $\mathrm{D}$ tôt

219

$7,8 \%$

5

$\mathrm{B}$ à $\mathrm{SP}$

150

$5,4 \%$

ताm

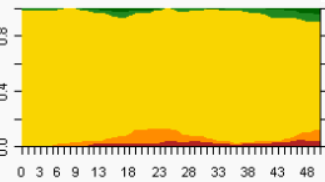

SP

160

$5,7 \%$

7

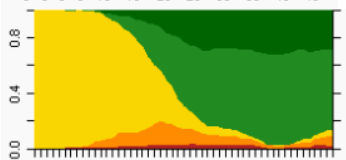

SP

160

SP à $\mathrm{B}$

258

$9,2 \%$

8

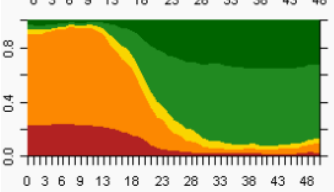

$\mathrm{D}$ à $\mathrm{B}$

373

$13,3 \%$

9

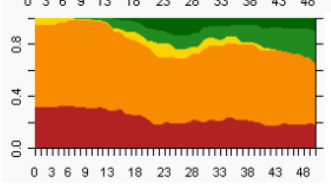

D

120

$4,3 \%$

10

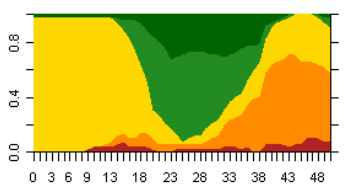

SP-B-D

52

$1,9 \%$

11

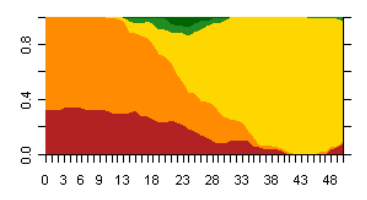

$\mathrm{D}$ à SP

66

$2,4 \%$

12

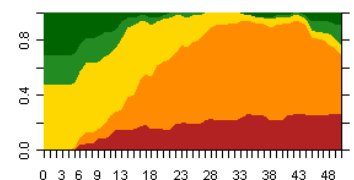

SP à D (très

tôt)

65

$2,3 \%$

Total

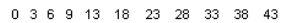

2799

$100 \%$

Source : INED, Enquête « Biographies et entourage », 2001.

Champ : Ensemble des enquêtés ayant répondu à la question $(\mathrm{N}=2799$ femmes et hommes Franciliens âgés de 50 à 70 ans).

Légende: $\square$ TD $\square$ D $\square$ SP $\square$ B $\square$ TB

Néanmoins, du fait des effectifs et afin de consolider nos analyses avec des statistiques multivariées, nous avons intérêt à réduire le nombre des classes. Ainsi, c'est à partir d'une typologie en 5 classes que nous allons approfondir l'analyse des caractéristiques des parcours 
de bien-être. Le tableau 5 présente la nature et la répartition des cinq types de trajectoires distinguées. Il apparaît clairement que, bien que d'effectifs plus réduits, deux types de trajectoires difficiles se distinguent toujours : les parcours globalement difficiles et ceux au long desquels la situation se détériore (classes 4 et 5 , soit 16,7 pourcents de l'ensemble des trajectoires perçues). De même, les trajectoires déclarées uniformément d'une tonalité neutre persistent à côté des trajectoires qui s'améliorent et de la classe prédominante des parcours globalement heureux (48 pourcents).

Tableau 5. Typologie de trajectoires de bien-être obtenues à partir d'une analyse de séquences

\begin{tabular}{lllll}
\hline numéro & Profil & N & $\%$ \\
1 & $\begin{array}{l}\text { Les parcours sans problème } \\
\text { Les parcours où la situation D à B }\end{array}$ & 362 & $12,9 \%$ \\
2 & $\begin{array}{l}\text { s'arrange } \\
\text { Les parcours globalement B }\end{array}$ & 1336 & $22,5 \%$ \\
3 & $\begin{array}{l}\text { heureux } \\
\text { Les parcours globalement D }\end{array}$ & 186 & $47,7 \%$ \\
4 & $\begin{array}{l}\text { difficiles } \\
\text { Les parcours où la situation se B à D }\end{array}$ & 284 & $6,6 \%$ \\
Total & détériore & 2799 & $10,1 \%$ \\
\hline
\end{tabular}

Source : INED, Enquête « Biographies et entourage », 2001.

Une façon de représenter les classes de la typologie consiste à construire des chronogrammes (Figure 3), i.e. des successions de coupes instantanées qui indiquent à chaque âge la distribution des individus de la classe entre les différents états. Autrement dit, à chaque moment de la trajectoire, le graphique présente les proportions cumulées d'individus dans chacune des situations. Bien que cette représentation occulte la dimension individuelle des trajectoires et masque l'hétérogénéité des parcours formant chaque type, elle offre une synthèse très parlante.

Reste désormais à identifier la façon dont ces classes se caractérisent du point de vue des individus qui les composent (les parcours difficiles sont-ils plus fréquents dans certaines catégories de la population ?) et à vérifier si les trajectoires perçues reflètent des histoires de vie particulières, marquées par des événements spécifiques. 

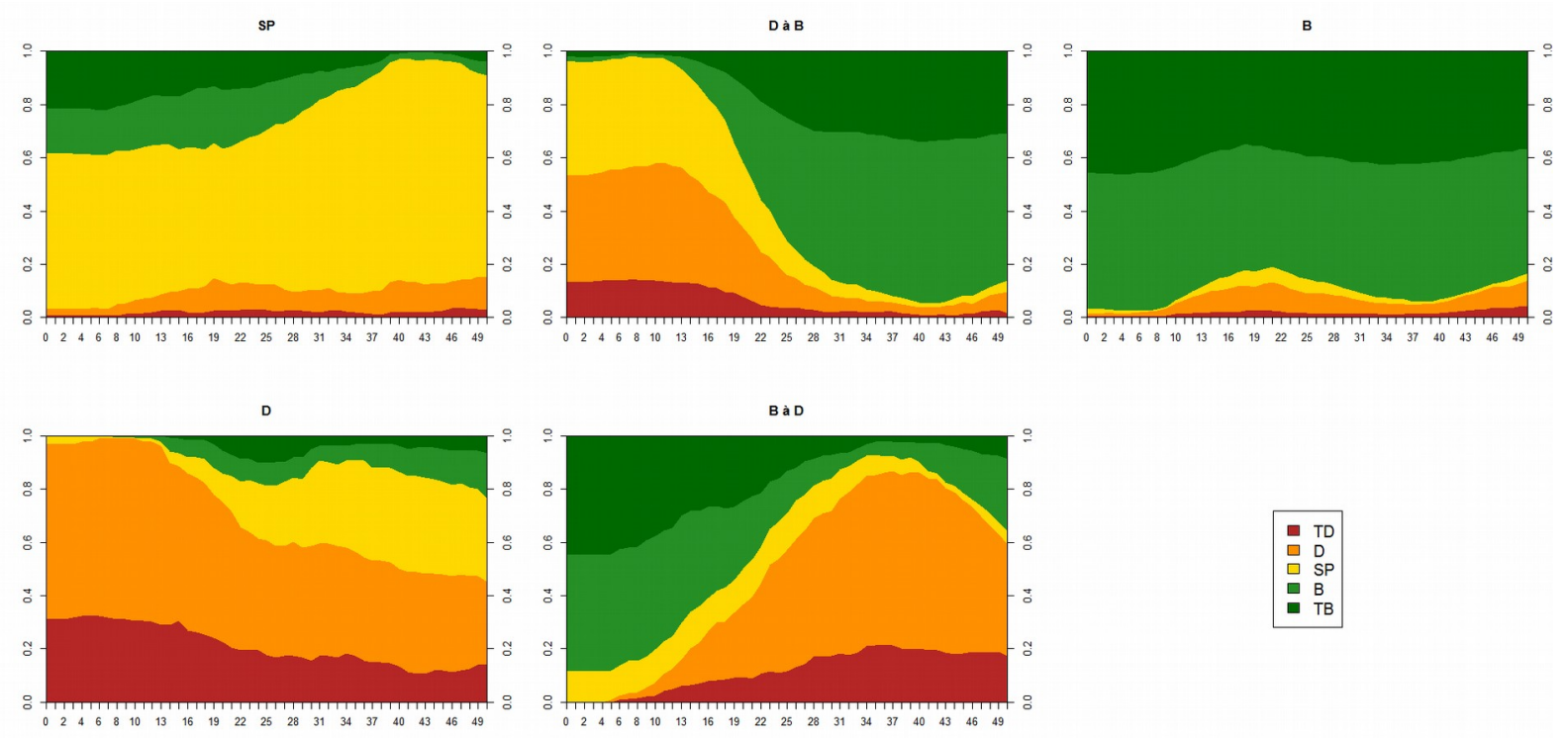

Source : INED, Enquête « Biographies et entourage », 2001.

Figure 3. Chronogrammes de la classification des trajectoires de bien-être

\section{Caractériser les types de trajectoires de bien-être perçu}

Afin de caractériser les cinq types de parcours, nous avons croisé la typologie en 5 classes avec deux types de critères : les caractéristiques socio-démographiques des individus d'une part ; les caractéristiques des parcours eux-mêmes d'autres part ${ }^{11}$.

\section{Une répartition différente selon les caractéristiques individuelles...}

Si les parcours globalement heureux dominent partout, c'est plus le cas pour les personnes nées pendant la guerre (1939-45). Les générations du baby-boom observées dans cette enquête (nées entre 1946 et 1950) déclarent plus fréquemment des trajectoires sans problème ainsi que des trajectoires qui se dégradent, dénotant des difficultés qui culminent à la trentaine au moment de la formation de la famille, comme si le passage d'un modèle traditionnel vers un modèle préconisé par l'émancipation avait été jugé a posteriori comme difficile (Bonvalet et al., 2011).

Les hommes et les femmes ne jugent pas le déroulement de l'existence sur les mêmes critères et ne traversent pas les mêmes épreuves, ce que traduisent des appréciations différenciées, sans que l'on puisse faire la part des faits et des perceptions. Si trois quarts des hommes et des femmes perçoivent leur parcours comme plutôt heureux (classes 2 et 3), les femmes sont plus nombreuses à le juger sans problème (15 pourcents versus 11 pourcents) et les hommes moins nombreux à juger que leurs circonstances se sont dégradées (type $5=8$ pourcents versus 12 pourcents pour les femmes).

Les différences selon le groupe socio-professionnel sont très significatives. Plus de la moitié des artisans, cadres et professions intermédiaires à 50 ans déclarent a posteriori une trajectoire de bien-être globalement heureuse (jusqu'à 55 pourcents parmi les cadres), contre seulement 40 pourcents des employés et ouvriers. De plus, 11 pourcents des parcours des ouvriers sont perçus comme totalement difficiles, quand c'est seulement le cas d'environ 4

\footnotetext{
${ }^{11}$ Les paragraphes qui suivent sont issus de l'analyse de tableaux croisés. Seules les associations significatives au seuil de 5 pourcents sont commentées (tests du khi-deux).
} 
pourcents des cadres et des artisans.

Une nette gradation s'observe dans la proportion des parcours jugés globalement heureux selon le niveau de diplôme, de seulement 34 pourcents pour les non-diplômés jusqu'à 54 pourcents pour ceux qui ont suivi des études supérieures. Les non-diplômés se distinguent d'ailleurs assez nettement du reste de la population : ils jugent leur parcours sans problème dans 15 pourcents des cas (versus 10 pourcents pour les personnes ayant au moins le baccalauréat); difficile ou s'étant détérioré dans 26 pourcents des cas (versus 15 pourcents pour les diplômés).

... et les trajectoires biographiques

Le type de trajectoire perçue est lié au lieu de naissance dans le sens où les parcours marqués par une migration de longue distance vers la métropole se distinguent : 11 pourcents des parcours des individus nés dans les Dom ou à l'étranger sont jugés globalement difficiles contre 5,5 pourcents pour ceux nés en Métropole.

Avoir déclaré une seule union est associé à une sur-représentation des parcours globalement heureux ou qui s'améliorent. A l'inverse, avoir connu un veuvage ou une séparation augmente la probabilité de déclarer un parcours difficile, i.e. d'appartenir aux classes 4 et 5 (respectivement 28 pourcents et 24 pourcents, versus 14 pourcents en l'absence de ces événements).

La taille de la famille n'a pas une influence univoque sur le fait de déclarer une trajectoire globalement heureuse : être seul ou avoir plus de 3 frères et sœurs résultent en des déclarations plus mitigées, et le rang dans la fratrie est peu significatif.

Le décès d'un parent durant l'enfance renforce la probabilité d'appartenir à la classe 2, celle des parcours qui s'améliorent ( 31 pourcents si c'est le décès du père, 39 pourcents si c'est celui de la mère, versus 22 pourcents en l'absence de décès).

Avoir connu une période de maladie double pratiquement la part des parcours difficiles mais également celles des parcours perçus comme alternant de bien à difficile ou de difficile à bien.

\section{Conclusion}

Disposant de reconstitutions factuelles de trajectoires individuelles d'un échantillon représentatif de Franciliens âgés de 50 à 70 ans, doublées de leur appréciation subjective, nous avons procédé à une analyse de ces trajectoires de bien-être perçu. De fait, le recueil et l'analyse quantitative de données de perception, déjà courantes dans d'autres disciplines et qui ont été largement documentées dans l'approche qualitative des histoires de vie, offrent un champ prometteur en analyse démographique des biographies. Nos travaux précédents ayant insisté sur le fait que la dichotomie classique entre données objectives et information subjective est - au moins partiellement - factice (GRAB, 2006), notre démarche se propose d'intégrer au mieux ces éléments, pour produire une analyse longitudinale des trajectoires de bien-être au long de la vie.

Le découpage de la vie en période fait apparaître un certain nombre de régularités, en termes de répartition par âge des «tournants biographiques" et de coïncidence entre événements factuels et tournants. De façon globale, l'analyse des caractérisations que donne chaque enquêté des différentes périodes de sa vie montre qu'en dépit des alternances entre périodes heureuses et moments difficiles, les trajectoires se déroulent dans leur majorité sur une tonalité positive. Ces premières explorations soulignent également le fait que les trajectoires se structurent fortement autour des événements familiaux, professionnels et migratoires, la vie conjugale occupant une place prééminente. 
D'un point de vue méthodologique, elles montrent qu'une telle approche - confrontant faits et perceptions - est réalisable dans le cadre d'opérations de collecte quantitatives et contribue à réinterroger les frontières entre les démarches qualitatives et quantitatives.

\section{Références}

Abbott A (1997) On the concept of turning point. Comparative Social Research 16 : 85-105.

Amiel MH, Godefroy P and Lollivier S (2013) Qualité de vie et bien-être vont souvent de pair. Insee Première 1428.

Bonvalet C, Clément C and Ogg J (2011) Réinventer la famille. L'histoire des baby-boomers. Paris: Presses universitaires de France.

Bonvalet C and Lelièvre E (2012) De la famille à l'entourage. Paris : INED - Presses universitaires de France.

Courgeau D (1985) Effet de déclarations erronées sur une analyse de données migratoires. In Poulain M (ed.) Migrations internes. Collecte des données et méthodes d'analyse. Cabay: Louvain-la-Neuve, 150-55.

Courgeau D and Lelièvre E (1989) Manuel d'analyse démographique des biographies. Paris: Presses universitaires de France.

Ferriss AL (2001) The domains of the quality of life. Bulletin de Méthodologie Sociologique $72: 5-19$.

Gabadinho A, Ritschard G, Müller NS and Studer M (2011) Analyzing and Visualizing State Sequences in R with TraMineR. Journal of Statistical Software 40(4): 1-37.

Golaz V and Lelièvre E (2012) L'entourage familial pendant l'enfance et l'adolescence, entre faits et perceptions. Une analyse rétrospective des parcours de vie des Franciliens des générations 1930-1950. Population-F 67(3): 491-516.

GRAB [Groupe de Réflexion sur l'Approche Biographique] (1999, 2006, 2010) Biographies d'enquêtes - Bilan de 14 collectes biographiques. Paris: INED.

Grossetti M (2006) L'imprévisibilité dans les parcours sociaux. Cahiers Internationaux de Sociologie $120: 5-28$.

Haller M and Müller B (2008) Characteristics of Personality and Identity in Population Surveys: Approaches for Operationalising and Localizing Variables to Explain Life Satisfaction. Bulletin de Méthodologie Sociologique 99: 5-33.

Hareven KT and Masaoka K (1988) Turning Points and Transitions - Perceptions of the Life Course. Journal of Family History 13(3): 271-89.

Jylhä M (2009) What is Self-Rated Health and Why Does It Predict Mortality? Towards a Unified Conceptual Model. Social Science and Medecine 69: 307-16.

Laborde C, Lelièvre E and Vivier G (2007) Trajectoires et événements marquants, comment dire sa vie ? Analyse des faits et des perceptions biographiques. Population-F 62(3): 567-86.

Lelièvre E and Vivier G (2001) Evaluation d'une collecte à la croisée du quantitatif et du qualitatif - L'enquête biographies et entourage. Population 56(6): 1043-73.

Lelièvre E, Roubaud F, Tichit C and Vivier G (2006) Données factuelles et perceptions, la place du flou dans l'observation et l'analyse. In Lelièvre E and Antoine P (eds) États flous et trajectoires complexes - Observation, modélisation, interprétation. Paris: INED/CEPED, 29-59.

Lesnard L and de Saint Pol T (2006) Introduction aux méthodes d'appariement optimal (Optimal Matching Analysis). Bulletin de Méthodologie Sociologique 90(1): 5-25. 
Macindoe H and Abbott A (2004) Sequence Analysis and Optimal Matching Techniques for Social Science Data. In Hardy M and Bryman A (eds) Handbook of Data Analysis. London: Sage, 387-406.

Mazuy M and Lelièvre E (2005) Déclarer ses enfants, déclarer ses conjoints - Rationalité des lecteurs et mode de questionnement. In Lefèvre $\mathrm{C}$ et Filhon $\mathrm{A}$ (eds) Histoires de familles, Histoires familiales. Paris: Cahiers de l'INED, 573-88.

Poulain M, Riandey B and Firdion M (1991) Enquête biographique et registre belge de population - Une confrontation des données. Population 46(1): 65-88.

Régnier-Loilier A (2007) Avoir des enfants en France - Désirs et réalités. Paris: Presses universitaires de France.

Robette N (2011) Explorer et décrire les parcours de vie - Les typologies de trajectoires. Paris: CEPED.

Annexe 1. Coïncidence entre jalons factuels et tournants biographiques selon le milieu social

\begin{tabular}{|c|c|c|c|c|c|c|}
\hline & $\begin{array}{l}\text { PCS } \\
\text { agriculteurs }\end{array}$ & $\begin{array}{l}\text { artisans } \\
\text { etc }\end{array}$ & cadres & $\begin{array}{l}\text { prof } \\
\text { int. }\end{array}$ & employés & ouvriers \\
\hline \multicolumn{7}{|l|}{ FAMILLE } \\
\hline Mariage & 52.5 & 41.6 & 36.6 & 44.2 & 51.5 & 37 \\
\hline Rang=1 & 54 & 43.8 & 39.1 & 45.9 & 54.8 & 39.7 \\
\hline Rang $>1$ & 36.1 & 12.5 & 11.5 & 25.9 & 21.7 & 17.9 \\
\hline Mise en couple & 51.4 & 42.9 & 35.4 & 42.3 & 50 & 40.3 \\
\hline Rang $=1$ & 54.1 & 43.5 & 39 & 46.9 & 54.7 & 44.1 \\
\hline Rang $>1$ & 37.8 & 40 & 25.7 & 25.9 & 30.7 & 26.3 \\
\hline $\begin{array}{l}\text { Séparation ou } \\
\text { divorce }\end{array}$ & 26.2 & 20 & 19.5 & 26 & 27.1 & 26 \\
\hline $\begin{array}{l}\text { Naissance d'un } \\
\text { enfant }\end{array}$ & 9.8 & 7.6 & 11.4 & 10.9 & 11.5 & 8.4 \\
\hline Rang=1 & 17.4 & 12.6 & 14.6 & 16.9 & 18.9 & 15.4 \\
\hline Rang $>1$ & 5.4 & 3.3 & 8.9 & 5.9 & 6.3 & 4.6 \\
\hline $\begin{array}{ll}\text { Naissance d'un } \\
\text { petit-enfant }\end{array}$ & 5.4 & 7 & 5.9 & 4.8 & 4.1 & 5.1 \\
\hline Rang $=1$ & 4.2 & 0 & 3.5 & 3.3 & 4.8 & 7.5 \\
\hline Rang $>1$ & 6.4 & 15.8 & 9.1 & 6.7 & 3.4 & 3.1 \\
\hline $\begin{array}{l}\text { Décès d'un } \\
\text { conjoint }\end{array}$ & 34.5 & 33.3 & 30.8 & 32.5 & 32.7 & 50 \\
\hline $\begin{array}{ll}\begin{array}{l}\text { Décès } \\
\text { père/mère }\end{array} & \mathrm{du}\end{array}$ & 12.4 & 12.3 & 10.3 & 9.8 & 11 & 9 \\
\hline Décès d'un enfant & 17.2 & 20 & 23.5 & 28 & 32.4 & 12.5 \\
\hline \multicolumn{7}{|c|}{\begin{tabular}{l|l} 
LOGEMENT &
\end{tabular}} \\
\hline $\begin{array}{l}\text { Changement de } \\
\text { logement }\end{array}$ & 20.8 & 22.5 & 21.4 & 21.2 & 22.1 & 23.5 \\
\hline $\begin{array}{l}\text { Accès à un } \\
\text { logement } \\
\text { indépendant }\end{array}$ & 22.1 & 22.9 & 21.5 & 22.2 & 23 & 22.5 \\
\hline Rang $=1$ & 48.3 & 46.7 & 38.1 & 41.3 & 48 & 42 \\
\hline Rang $>1$ & 13.9 & 14.8 & 17.2 & 16.3 & 14.9 & 16.1 \\
\hline Accession à la & 15.6 & 18.8 & 14.5 & 14.6 & 13.6 & 17.5 \\
\hline
\end{tabular}




\begin{tabular}{l|llllll}
$\begin{array}{l}\text { propriété } \\
\text { Rang=1 }\end{array}$ & 18.8 & 21 & 16.6 & 17.1 & 15.4 & 19.3 \\
Rang>1 & 10.4 & 14.9 & 11.6 & 10.3 & 10.6 & 11.1 \\
\hline TRAVAIL & & & & & & \\
Changement & 20.2 & 23.1 & 20.8 & 19 & 18.4 & 21.6 \\
d'emploi & 29.4 & 36.7 & 38.1 & 27.9 & 28.5 & 31.4 \\
Rang=1 & 16.6 & 19.1 & 16.6 & 16.7 & 15.3 & 19.2 \\
Rang>1 & 11.4 & 0 & 21.6 & 20.3 & 19.3 & 28.6 \\
Chômage & 11.1 & 50 & 31.4 & 24.1 & 26.8 & 17.6 \\
Reprise d'études & 11.1 & 50.6 & & &
\end{tabular}

Source : INED, Enquête « Biographies et entourage », 2001.

Champ : Ensemble des enquêtés ayant répondu à la question $(\mathrm{N}=2799$ femmes et hommes Franciliens âgés de 50 à 70 ans).

Lecture : 44,3 pourcent des mariages coïncident (à un an près) avec un tournant biographique.

\section{Annexe 2. L'appariement optimal}

L'appariement optimal (Optimal Matching Analysis) est une technique statistique apparue dans les années 1950. Utilisée notamment en biologie pour l'étude de l'ADN, elle est introduite dans le champ des sciences sociales par le sociologue Andrew Abbott (Abbott et Forrest, 1986). Son principe consiste à mesurer la dissimilarité entre deux séquences sous la forme du coût de transformation de l'une des séquences en l'autre (Lesnard et de Saint Pol, 2006). La transformation est effectuée au moyen de trois opérations élémentaires : l'insertion d'un élément dans une séquence ; la suppression d'un élément et la substitution d'un élément par un autre. À chacune de ces opérations élémentaires est associé un coût. À partir de ces opérations élémentaires, il existe plusieurs manières de transformer une séquence en une autre : la mesure de la dissimilarité entre les séquences est alors égale au coût minimal de transformation de l'une en l'autre. Le calcul de la dissimilarité entre l'ensemble des paires de séquences mène à la création d'une matrice de distance entre les séquences, qui peut être utilisée pour des analyses ultérieures telles qu'une classification automatique.

Dans notre application, l'univers des cinq états possibles est hiérarchisé : de «Très Difficile » à « Très Bien », en passant par « Difficile », « Sans Problème » et « Bien ». Il peut donc sembler souhaitable de tenir compte de la proximité de deux états donnés lorsque l'on fixe leur coût de substitution, i.e. deux états proches dans la hiérarchie sont considérés comme relativement similaire et leur coût de substitution choisi est faible. La comparaison de différents paramétrages nous a finalement conduits à conserver la matrice de coûts suivante, qui permet d'obtenir une partition en classes homogènes et pertinentes :

\begin{tabular}{|c|c|c|c|c|c}
\hline & TD & D & SP & B & TB \\
\hline TD & 0 & 0 & 1 & 2 & 2 \\
\hline D & 0 & 0 & 1 & 2 & 2 \\
\hline SP & 1 & 1 & 0 & 1 & 1 \\
\hline B & 2 & 2 & 1 & 0 & 0 \\
\hline TB & 2 & 2 & 1 & 0 & 0 \\
\hline
\end{tabular}

Dans la mesure où il nous semblait important de prendre en compte l'âge des changements de tonalité dans la trajectoire perçue, le coût d'insertion et de suppression est de 1,1 , soit une valeur légèrement supérieure à la moitié du coût de substitution maximal : cela permet de 
limiter l'usage des opérations d'insertion et de suppression et donc de conserver l'information sur le moment auquel interviennent les événements qui scandent les trajectoires (Macindoe et Abbott, 2004).

Ces analyses ont été effectuées à partir du package TraMineR du logiciel $\mathrm{R}$ (Gabadinho et al., 2011). 